\title{
Educação permanente em saúde: concepções e proposições no Projeto de formação e melhoria da qualidade de rede de atenção à saúde (QualiSUS-Rede)
}

Permanent Education in Health: concepts and propositions in the Project Training and Improvement of Quality of Health Care Network (QualiSUS-Network) Formación Permanente de Salud: conceptos y proposiciones en la Formación y Mejora de la Calidad del Proyecto de Red de Servicios Médicos (QualiSUS-Net)

\author{
Patrícia Pássaro da Silva TOLEDO ${ }^{(1)}$ \\ Marly Marques da CRUZ ${ }^{(1)}$ \\ Rafaela Barros Chagas de SOUZA ${ }^{(1)}$
}

Recebido: 11 jul 2017 Revisado: 23 ago 2017 Aceito: 26 ago 2017

\section{Autor de}

correspondência: Patrícia Pássaro da Silva Toledo

e-mail:

patriciapassaro@gmail.com

Conflito de interesses: Os autores declaram não haver nenhum interesse profissional ou pessoal que possa gerar conflito de interesses em relação a este manuscrito.

\section{Resumo}

O objetivo do estudo foi analisar as propostas de educação permanente em saúde - EPS no Projeto de Formação e Melhoria da Qualidade de Rede de Atenção à Saúde - QualiSUSRede visando identificar se estas ações estão alinhadas com o conceito de EPS da literatura. Delineou-se uma pesquisa qualitativa, descritiva e exploratória, utilizando a base documental e os dados secundários dos subprojetos QualiSUS-Rede. Dentre os quinze subprojetos, foram selecionados três que englobavam ações educativas que se repetiam nos demais. As 33 ações propostas foram listadas e classificadas de acordo com sua temática e tipos de atividades, sendo posteriormente classificadas, de acordo com as suas características, como EPS ou como Educação Continuada - EC. Das atividades analisadas, apenas $15,2 \%$ estavam alinhadas as concepções de EPS enquanto $84,8 \%$ se referiam a atividades características de EC. Quanto à temática, 33\% tratava diretamente das redes de atenção à saúde - RAS e $64 \%$ abordavam temas relacionados indiretamente a RAS. Os dados demonstram que as propostas educativas dos subprojetos não estão, em sua maioria, alinhadas aos conceitos de EPS, indicando a existência de uma lacuna entre as concepções de EPS como política pública de saúde e a compreensão dos profissionais e gestores do Sistema Único de Saúde.

Descritores: Educação em Saúde; Educação Permanente; Educação Continuada; Atenção à Saúde; Melhoria da Qualidade. 


\begin{abstract}
This study analyzed the proposals for Permanent Education in Health (PEH) in the Project of Training and Improvement of Quality of Health Care Network (QualiSUS-Network) in order to identify whether these actions are aligned with the concept of PEH literature. It outlined a qualitative, descriptive and exploratory research, using documental analyze and secondary data QualiSUS-Network subprojects. Among the fifteen subprojects were selected three that encompassed educational activities that were repeated in others. The 33 proposed actions have been listed and classified according to their themes and types of activities, subsequently classified according to their characteristics, such as PEH or as Continuing Education (CE). The activities analyzed, only $15.2 \%$ were lined up the PEH conceptions while $84.8 \%$ referred the CE characteristics activities. Regarding the theme, 33\% directly dealt with the health care networks $(\mathrm{HCN})$ and $64 \%$ indirectly addressed issues related to HCN. The results demonstrate that the educational proposals of the subprojects are not, mostly, aligned with concepts of $\mathrm{PEH}$, indicating the existence of a gap between the PEH concepts as public health policy and understanding of professionals and managers of Brazilian health system (SUS).
\end{abstract}

Keywords: Health Education; Professional Education; Continuing Education, Health Care; Quality Improvement.

\begin{abstract}
Resumen
El objetivo del estudio fue analizar las propuestas de educación permanente en salud (EPS) en el Proyecto de Formación y Mejora de la Calidad de Red de Atención a la Salud (QualiSUS-Red) para identificar si estas acciones están alineadas con el concepto de EPS de la literatura. Se elaboró una investigación cualitativa, descriptiva y exploratoria, utilizando la base documental y los datos secundarios de los subproyectos QualiSUS-Red. Entre los quince subproyectos, fueron seleccionados tres que englobaban acciones educativas que se repetían en los demás. Las 33 acciones propuestas fueron listadas y clasificadas de acuerdo con su temática y tipos de actividades, siendo posteriormente clasificadas, de acuerdo con sus características, como EPS o como Educación Continua (EC). De las actividades analizadas, sólo el 15,2\% estaban alineadas a las concepciones de EPS mientras que el $84,8 \%$ se refería a actividades características de EC. En cuanto a la temática, el $33 \%$ trataba directamente de las redes de atención a la salud (RAS) y el 64\% abordaba temas relacionados indirectamente con RAS. Los datos demuestran que las propuestas educativas de los subproyectos no están, en su mayoría, alineadas a los conceptos de EPS, indicando la existencia de una brecha entre las concepciones de EPS como política pública de salud y la comprensión de los profesionales y gestores del Sistema Único de Salud.
\end{abstract}

Palabras-claves: Educación en Salud; Educación Permanente; Educación Continuada; Atención a la Salud; Mejora de la Calidad.

\title{
Introdução
}

\author{
A interseção entre as áreas da Educação e da Saúde ocorre a partir da necessidade \\ de orientar e organizar a formação de recursos humanos e o desenvolvimento de \\ trabalhadores da saúde para uma atuação em defesa do caráter público, universal, integral, \\ equânime e democrático do Sistema Único de Saúde - SUS. É neste contexto que a
}


ordenação da formação em saúde passa a ser de responsabilidade do SUS e, dois campos com objetivos convergentes e práticas distintas, emergem: a educação em saúde e a educação na saúde. ${ }^{1}$

A educação em saúde surge em 1908, nos EUA, como uma estratégia de prevenção de doenças ${ }^{1-4}$ e hoje se apoia na compreensão da educação como um mecanismo estratégico para subsidiar o desenvolvimento do indivíduo e da coletividade, de maneira a incitar a reflexão e a consciência crítica, o exercício da cidadania e da autonomia, permitindo desta forma a intervenção e a transformação das condições de saúde e doença, empoderando o indivíduo como um ator político e socialmente ativo. ${ }^{1-4}$ Ela não se refere à profissionalização, mas sim a um processo educativo que visa à construção de conhecimentos em saúde para a população, contribuindo para o cuidado em saúde. ${ }^{1}$

A educação na saúde, por sua vez, está relacionada à "produção e sistematização de conhecimentos relativos à formação e ao desenvolvimento para a atuação em saúde, envolvendo práticas de ensino, diretrizes didáticas e orientação curricular" ${ }^{1,}$ p. 20 Nesse sentido está diretamente orientada para a dimensão do trabalho, suas especificidades, suas práticas, seus processos e a utilização de tecnologias em sua produção. Propõe-se a ordenar a formação em saúde (nível técnico, graduação e pós-graduação) e contemplar o desenvolvimento dos profissionais de saúde por meio da educação continuada - EC e da educação permanente em saúde - EPS.

A EC emerge da necessidade de capacitação de trabalhadores da saúde inseridos nos serviços e se refere ao "processo de aquisição sequencial e acumulativa de informações técnico-científicas pelo trabalhador, por meio de escolarização formal, de vivências, de experiências laborais e de participação no âmbito institucional ou fora dele". 1, p. 19 Ela prioriza a transmissão dos saberes através de treinamentos e capacitações com foco nas categorias profissionais e nas necessidades de conhecimento técnico-científico de cada área. ${ }^{1} \mathrm{~A}$ EPS, por sua vez, coloca o processo de trabalho como eixo fundamental para a aprendizagem, ou seja, procura alternativas e soluções para questões reais e concretas do trabalho em 
saúde, privilegiando a criatividade humana, as discussões e análises participativas dos problemas vivenciados e as possíveis causas e efeitos que estas alternativas de solução podem representar na transformação das práticas de saúde. ${ }^{1}$

O trabalho é valorizado como fonte de saberes pela EPS, que coloca o processo de trabalho cotidiano como alicerce para produção de conhecimento e desenvolvimento de ações educativas contextualizadas, participativas e interdisciplinares, sempre articulando três dimensões: atenção à saúde, gestão e controle social. ${ }^{10}$ Para Montanha e Peduzzi ${ }^{12}$ a EPS se institui como um projeto político pedagógico, estabelecido por ações e reflexões sobre o cotidiano dos serviços de saúde e a realidade vivenciada pelos trabalhadores, demandando, obrigatoriamente, uma interação entre a teoria e prática com uma abordagem interdisciplinar e multiprofissional. O seu objetivo é a transformação das práticas profissionais, das práticas de saúde e da própria organização do trabalho em saúde, buscando a integralidade do trabalho multiprofissional, a garantia e a ampliação da autonomia e da cidadania dos trabalhadores e dos usuários. ${ }^{13}$ Nesta perspectiva a EPS se distancia da educação tradicional, pois não se apresenta como reprodutora de conceitos ou práticas já instituídas.

Sendo assim, para Ceccim e Feuerwerker ${ }^{14}$ a grande diferença é que enquanto a EC se apodera do cenário de práticas como território de aplicação dos conhecimentos, a EPS se utiliza deste cenário de práticas como território de aprendizagem a partir do momento em que informa e recria a teoria necessária, recriando a sua própria prática. Na visão desses autores, a EPS deve objetivar a aproximação dos profissionais às necessidades coletivas de saúde a partir da busca por alternativas, recursos e soluções para os problemas de saúde reais, devendo, portanto, ser compreendida como um instrumento de transformação das práticas em saúde através de um processo crítico e reflexivo, que necessita de retroalimentação constante. ${ }^{14}$

A primeira Política Nacional de Educação Permanente em Saúde - PNEPS no Brasil foi criada pela Portaria no 198/GM de $2004^{15}$ que buscava valorizar justamente essa integração 
e articulação entre o ensino, a gestão, as ações em saúde e o controle social. Na sequência a Portaria $n^{\circ} 1.996 / G M$ de $2007^{16}$ implementou a nova PNEPS que dispõe, dentre outras questões, sobre a necessidade de considerar as especificidades de cada região, superar as desigualdades e atender as demandas de formação e desenvolvimento para o trabalho em saúde.

Dentro desta perspectiva de ordenamento da formação de recursos humanos e de desenvolvimento dos trabalhadores do SUS, em 2011, a Secretaria de Gestão do Trabalho e da Educação na Saúde - SGTES consolidou em um documento todas as suas ações, políticas e programas que visavam fomentar a "formação, educação permanente, valorização dos trabalhadores e democratização das relações de trabalho no SUS". 17, p. 7 Tal documento tem sido norteador das diversas ações no campo da formação profissional em saúde e entendese que as propostas educativas elaboradas pelos profissionais responsáveis, geralmente gestores e técnicos, representam seu entendimento quanto ao conceito de educação permanente expressando as necessidades pungentes dos serviços de saúde.

Neste sentido, este estudo buscou compreender as concepções de EPS presente nos subprojetos vinculados ao Projeto de Formação e Melhoria da Qualidade de Rede de Atenção à Saúde (QualiSUS-Rede) com a finalidade de identificar se as ações educativas propostas estão alinhadas com o conceito de EPS. Desta forma foi possível traçar um panorama da compreensão dos profissionais de saúde em relação à concepção de EPS, especialmente daqueles em cargo de gestão e técnicos responsáveis pelo planejamento de atividades educativas.

\section{Metodologia}

A pesquisa realizada é classificada como qualitativa, descritiva e exploratória por se tratar de um tema recente no âmbito das políticas de saúde no Brasil. Daí abordar a temática por meio da base conceitual e documental referente a educação permanente em saúde 
considerando suas concepções e o delineamento de ações numa etapa de planejamento dos projetos do QualiSUS-Rede.

As fontes bibliográficas foram utilizadas para se chegar as concepções de educação permanente. Por outro lado, a fonte de dados secundários e a apreciação documental foram utilizadas para analisar as propostas construídas sobre formação/educação na saúde no âmbito do projeto QualiSUS-Rede. Deste modo foram analisados o Manual Operacional do Projeto QualiSUS-Rede ${ }^{18}$ e os respectivos subprojetos.

Como forma de acompanhamento da implementação dos subprojetos, o Projeto QualiSUS-Rede previa, em sua etapa 3, "a formulação e implementação do programa de acompanhamento, monitoramento e avaliação". ${ }^{18, ~ p . ~} 40$ Para tanto, foi desenvolvido pela Unidade de Gestão do Projeto QualiSUS-Rede (UGP/DESID/SE/MS) em parceria com o Laboratório de Avaliação de Situações Endêmicas Regionais - LASER/DENSP/FIOCRUZ um Projeto de Avaliação de Implementação do Projeto de Formação e Melhoria da Qualidade da Rede de Atenção à Saúde - QualiSUS-Rede, chamado aqui de Estudo Avaliativo QualiSUS-Rede, visando não confundir os respectivos projetos (Projeto QualiSUS-Rede e Projeto de Avaliação do QualiSUS-Rede). ${ }^{18}$

Com os eixos estruturantes e objetivos estratégicos do Projeto QualiSUS-Rede definidos a equipe do Estudo Avaliativo QualiSUS-Rede, a UGP e os membros do Grupo Condutor - GC de cada subprojeto elaboraram um modelo lógico - ML com todas as ações propostas para cada eixo padronizado. Os ML visam facilitar a visualização da racionalidade da intervenção, ou seja, as ações dos subprojetos, as relações de dependência e consequência entre insumos e efeitos, descrevendo os fluxos e as interações entre as atividades e seus resultados esperados.

Para elaboração deste estudo foi realizada uma análise inicial dos quinze subprojetos e de seus respectivos ML com a finalidade de identificar em quais subprojetos foram propostas ações de educação em saúde. Todos os projetos continham propostas educativas. Assim, para seleção dos três subprojetos foram utilizados os seguintes critérios: amplitude 
das atividades educativas, no que se refere ao formato das ações propostas, ou seja, os subprojetos que abrangessem a maior diversidade de formas de operacionalização das atividades educativas; que fossem de regiões político-administrativas diferentes; e que contemplassem as propostas que se repetiam nos demais subprojetos, de modo que os escolhidos fossem representativos em termos de ações e temáticas. As propostas educativas elaboradas pelos gestores e técnicos que integram o GC de cada subprojeto regional e apresentadas nos respectivos ML nos permitiram uma aproximação com a compreensão destes profissionais em relação às concepções de EPS, às suas metodologias e à sua aplicabilidade de acordo com as temáticas sugeridas.

Os subprojetos selecionados foram identificados pelos números I, II e III para garantir seu anonimato. Por sua vez, as ações educativas propostas foram listadas e classificadas de acordo com sua metodologia e sua temática, sendo posteriormente classificadas como EC ou EPS em relação as suas características. Para tal utilizou-se de medidas de proporção para verificar a distribuição conforme categoria.

Este estudo foi aprovado pelo Comitê de Ética em Pesquisa em 16 de Janeiro de 2015 sob o número de parecer 933.894.

\section{Resultados e Discussão}

\section{O Projeto QualiSUS-Rede}

O Projeto QualiSUS - Rede surge da prioridade, assinalada pelo Ministério da Saúde - MS, de estruturação, organização e qualificação de redes integradas e regionalizadas de atenção à saúde. Toda a sua concepção foi fundamentada em estudos, pesquisas e ampla discussão acerca das fragilidades assistenciais, de gestão e de organização dos serviços de saúde no SUS. ${ }^{18}$ Sua finalidade era estimular e promover a consolidação de um sistema integrado de serviços de saúde, estruturado por meio de Redes de Atenção à Saúde - RAS, através de uma intervenção articulada entre o MS, estados e municípios, com objetivos 
claros, duração e recursos definidos. A pretensão era a de contribuir para a qualificação da atenção e da gestão em saúde, por meio da organização de redes regionais e temáticas de atenção à saúde, no âmbito do SUS. Seus objetivos principais eram a organização das RAS com ênfase no protagonismo da atenção primária para o seu ordenamento, com investimentos na atenção especializada e nos sistemas logísticos de suporte à rede, fortalecendo e aprimorando os mecanismos de gestão das RAS, a regionalização, a contratualização, a regulação do acesso, a responsabilização dos gestores e a participação social. ${ }^{19}$

O projeto se dedicou à implantação de quinze experiências de RAS, sendo dez experiências em Regiões de Saúde vinculadas às regiões metropolitanas - RM e cinco Regiões denominadas "Tipo" (caracterizadas conforme suas singularidades). As quinze regiões escolhidas contemplam a participação de 17 estados e do Distrito Federal, abarcando todas as regiões do país e contemplando a diversidade nacional e singularidades das regiões de saúde e suas bases territoriais. Cada região definiu o Grupo Condutor - GC, responsável pela formulação e implementação do Subprojeto, composto por um coordenador formalmente designado pela Secretaria Estadual de Saúde - SES e por componentes representantes da SES, representantes dos municípios envolvidos, representante do COSEMS, um Apoiador Local do MS e por representante das Instituições de Ensino e Pesquisa, em parceria, caso necessário.

Orientados pela Portaria $n^{\circ} 4.279$ de $2010,{ }^{20}$ aprovada pela Comissão Intergestores Tripatite - CIT, e pelo Decreto $n^{\circ} 7.508$ de 2011, ${ }^{21}$ e instituído pela Portaria $n^{\circ} 396$ de 2011, ${ }^{19}$ os GC das regiões selecionadas para o QualiSUS-Rede elaboraram Subprojetos de RAS norteados pelos cinco eixos estruturantes do Projeto: Eixo 1 - Atenção Primária em Saúde; Eixo 2 - Redes Temáticas (Rede de Atenção à Mulher e Criança, Rede de Urgência e Emergência, Rede de Atenção Psicossocial e Rede Câncer); Eixo 3 - Sistemas de Apoio Diagnóstico e Terapêutico - SADT; Eixo 4 - Sistema de Apoio Logístico; Eixo 5 Fortalecimento da Governança Regional. Foram também definidos os objetivos estratégicos, 
que são as atuações prioritárias e mais relevantes para a concretização dos eixos estruturantes.

As atividades de EPS encontram-se elencadas na área de financiamento do projeto como provisão de apoio para a qualificação da gestão integrada da RAS. Tal provisionamento considera ações de planejamento e implementação de um programa contínuo de qualificação para toda RAS e o desenvolvimento de programas de EPS que contemplem as estratégias de mudanças de gestão, de comportamento dos prestadores de serviços e de práticas clínicas. ${ }^{19}$ Estava previsto no Projeto a parceria e articulação com a Secretaria de Gestão do Trabalho e da Educação em Saúde - SGETS para a consolidação de ações que objetivassem fomentar a inclusão de discussões sobre as RAS nos ambientes de formação e capacitação, assinalando a importância de ações educativas planejadas e articuladas aos objetivos de consolidação e organização das RAS. ${ }^{18}$

Desta forma, todos os subprojetos deveriam conter, além das intervenções necessárias para organização das RAS, propostas de EPS sob a forma de ações educativas que estivessem alinhadas aos cinco eixos estruturantes do Projeto QualiSUS-Rede. Estas ações educativas poderiam estar articuladas com os Planos Estadual e/ou Regional de Educação Permanente em Saúde (PEEPS ou PREPS) e contemplar as necessidades de formação e qualificação dos profissionais de saúde.

\section{Modelos lógicos dos subprojetos do QualiSUS-Rede}

Os modelos lógicos - ML dos subprojetos analisados apresentavam as propostas elaboradas pelos membros do GC de cada região para cada Eixo Estruturante do Projeto e tais propostas contemplavam as mais variadas atividades, dentre elas, as ações educativas. Foram identificadas 33 propostas de ações educativas distribuídas nos três subprojetos selecionados, sendo sete ações educativas no Subprojeto I; 21 propostas no Subprojeto II; e cinco atividades educativas no Subprojeto III (Quadro 1). 
ISSN 2179-6750

\begin{tabular}{|c|c|}
\hline Subprojeto & Proposta de Ação Educativa \\
\hline \multirow{7}{*}{$\mathbf{I}$} & Curso modular em EAD com foco nas redes temáticas \\
\hline & $\begin{array}{c}\text { Capacitar profissionais em atendimento ao paciente psiquiátrico em situação de } \\
\text { emergência, nos pontos da RAPS e RUE }\end{array}$ \\
\hline & Capacitar profissionais dos pontos de atenção da RUE em ATLS, ACLS, APLS, ALSO \\
\hline & Capacitar profissionais do componente móvel da Urgência em PHLS pré- Hospitalar \\
\hline & $\begin{array}{l}\text { Realizar } 03 \text { oficinas de capacitação técnica para qualificação de profissionais de saúde na } \\
\text { rede psicossocial }\end{array}$ \\
\hline & Desenvolver cursos modulares em EAD com foco na rede psicossocial \\
\hline & $\begin{array}{l}\text { Realizar dois Seminários: um de introdução aos desafios da RAPS e outro para avaliação da } \\
\text { estruturação da rede. }\end{array}$ \\
\hline \multirow{17}{*}{ II } & Realizar 10 oficinas para adequar protocolos clínicos das linhas de cuidado. \\
\hline & Realizar oficinas para elaborar protocolos de regulação de acesso \\
\hline & $\begin{array}{c}\text { Realizar } 15 \text { cursos para capacitar } 375 \text { agentes de combate as endemias em ações de } \\
\text { prevenção e controle das doenças endêmicas }\end{array}$ \\
\hline & Realizar 15 cursos para 375 ACS nas linhas de cuidado prioritárias \\
\hline & $\begin{array}{c}\text { Realizar } 30 \text { treinamentos para } 500 \text { profissionais da rede de atenção nas ações de } \\
\text { planejamento familiar }\end{array}$ \\
\hline & Realizar 20 cursos de atualização para as equipes de saúde da família \\
\hline & Realizar 30 cursos de capacitação para os profissionais da rede de Atenção Básica no SIAB \\
\hline & Realizar 11 treinamentos para profissionais da rede de Atenção Básica em imunização \\
\hline & $\begin{array}{c}\text { Realizar } 10 \text { treinamentos para } 500 \text { profissionais de nível superior e médio das unidades fixas } \\
\text { e pré-hospitalares móveis da rede de U/E nos protocolos da RUE }\end{array}$ \\
\hline & Realizar 1 Curso Pós-Técnico em UTI pediátrica para 30 profissionais \\
\hline & Realizar 5 treinamentos para 150 enfermeiros na coleta de exame de colo de útero \\
\hline & Realizar Curso Pós-Técnico em Oncologia para 30 técnicos de enfermagem \\
\hline & Realizar Curso Pós-Técnico em Mamografia para 25 técnicos de radiologia \\
\hline & $\begin{array}{c}\text { Realizar } 5 \text { treinamentos para } 150 \text { profissionais no rastreamento e diagnóstico dos cânceres } \\
\text { mais prevalentes na região }\end{array}$ \\
\hline & Realizar curso de atualização em radioterapia para 25 técnicos de radiologia \\
\hline & $\begin{array}{l}\text { Realizar } 5 \text { treinamentos para } 100 \text { profissionais de saúde dos municípios para o uso da } \\
\text { tecnologia do Telessaúde }\end{array}$ \\
\hline & Capacitar os profissionais de saúde no SISREG \\
\hline
\end{tabular}




\begin{tabular}{|c|c|}
\hline & $\begin{array}{c}\text { Realizar } 2 \text { oficinas para } 60 \text { profissionais sobre o desenho das RAS instituídas com vistas aos } \\
\text { fluxos de referência e contra referência }\end{array}$ \\
\hline & Capacitar representantes da sociedade civil sobre concepções de RAS \\
\hline & $\begin{array}{c}\text { Capacitar coordenadores e apoiadores de rede e conselheiros municipais de saúde no } \\
\text { entendimento de organização das RAS }\end{array}$ \\
\hline & $\begin{array}{l}\text { Capacitar gestores e profissionais de planejamento no conceito de redes, instrumentos } \\
\text { legais e projetos vigentes }\end{array}$ \\
\hline \multirow{5}{*}{ III } & Capacitar profissionais da ESF para o diagnóstico da Retinopatia diabética \\
\hline & $\begin{array}{l}\text { Realizar capacitações dos profissionais (de acordo com as unidades especificadas) em } \\
\text { cursos de imersão; promover capacitação específica em telemonitoramento para } \\
\text { profissionais da assistência aos pacientes graves neonatais das UTIs da Rede Cegonha }\end{array}$ \\
\hline & Capacitar os profissionais dos serviços para utilizar o software de regulação \\
\hline & Treinar profissionais para trabalhar com transporte sanitário (ESTADO) \\
\hline & Realizar oficinas regionais para construção do documento de planejamento regional \\
\hline
\end{tabular}

Quadro 1. Ações educativas propostas pelos três subprojetos do QualiSUS-Rede

Fonte: Elaboração própria (2017).

As atividades educativas foram classificadas de acordo com sua temática, sendo categorizadas a partir dos assuntos abordados em cada ação. Estes temas foram agrupados em cinco categorias: Formação Técnica/Profissional; Estruturação das Redes de Atenção à Saúde; Planejamento das Redes de Atenção à Saúde; Sistemas de Informação e Telessaúde; e Elaboração de Protocolos (Quadro 2).

\section{Formação Técnica/Profissional - Nº de Ações: 20 (60,6\%)}

Capacitar profissionais em atendimento ao paciente psiquiátrico em situação de emergência, nos pontos da RAPS e RUE

Capacitar profissionais dos pontos de atenção da RUE em ATLS, ACLS, APLS, ALSO

Capacitar profissionais do componente móvel da Urgência em PHLS pré-Hospitalar

Realizar 03 oficinas de capacitação técnica para qualificação de profissionais de saúde na rede psicossocial 
Realizar 15 cursos para capacitar 375 agentes de combate as endemias em ações de prevenção e controle das doenças endêmicas

Realizar 10 treinamentos para 500 profissionais de nível superior e médio das unidades fixas e pré-hospitalares móveis da rede de U/E nos protocolos da RUE

Desenvolver cursos modulares em EAD com foco na rede psicossocial

Realizar 15 cursos para 375 ACS nas linhas de cuidado prioritárias

Realizar 30 treinamentos para 500 profissionais da rede de atenção nas ações de planejamento familiar

Realizar 20 cursos de atualização para as equipes de saúde da família

Realizar 11 treinamentos para profissionais da rede de Atenção Básica em imunização

Realizar 5 treinamentos para 150 profissionais no rastreamento e diagnóstico dos cânceres mais prevalentes na região

Realizar 1 Curso Pós-Técnico em UTI pediátrica para 30 profissionais

Realizar 5 treinamentos para 150 enfermeiros na coleta de exame de colo de útero

Realizar Curso Pós-Técnico em Oncologia para 30 técnicos de enfermagem

Realizar Curso Pós-Técnico em Mamografia para 25 técnicos de radiologia

Realizar capacitações dos profissionais (de acordo com as unidades especificadas) em cursos de imersão; promover capacitação específica em telemonitoramento para profissionais da assistência aos pacientes graves neonatais das UTIs da Rede Cegonha

Realizar curso de atualização em radioterapia para 25 técnicos de radiologia

Capacitar profissionais da ESF para o diagnóstico da Retinopatia diabética

Treinar profissionais para trabalhar com transporte sanitário (ESTADO)

Estruturação das Redes de Atenção à Saúde - RAS - Nº de Ações: 6 (18,2\%)

Realizar dois Seminários: um de introdução aos desafios da RAPS e outro para avaliação da estruturação da rede. 


\begin{tabular}{|c|}
\hline Curso modular em EAD com foco nas redes temáticas \\
\hline Capacitar representantes da sociedade civil sobre concepções de RAS \\
\hline $\begin{array}{l}\text { Realizar } 2 \text { oficinas para } 60 \text { profissionais sobre o desenho das RAS instituídas com vistas } \\
\text { aos fluxos de referência e contra referência }\end{array}$ \\
\hline $\begin{array}{l}\text { Capacitar coordenadores e apoiadores de rede e conselheiros municipais de saúde no } \\
\qquad \text { entendimento de organização das RAS }\end{array}$ \\
\hline $\begin{array}{l}\text { Capacitar gestores e profissionais de planejamento no conceito de redes, instrumentos } \\
\text { legais e projetos vigentes }\end{array}$ \\
\hline Sistemas de Informação e Telessaúde - No de Ações: 4 (12,1\%) \\
\hline Realizar 30 cursos de capacitação para os profissionais da rede de Atenção Básica no SIAB \\
\hline Capacitar os profissionais de saúde no SISREG \\
\hline Capacitar os profissionais dos serviços para utilizar o software de regulação \\
\hline $\begin{array}{l}\text { Realizar } 5 \text { treinamentos para } 100 \text { profissionais de saúde dos municípios para o uso da } \\
\text { tecnologia do Telessaúde }\end{array}$ \\
\hline Elaboração de Protocolos - Nº de Ações: $2(6,1 \%)$ \\
\hline Realizar 10 oficinas para adequar protocolos clínicos das linhas de cuidado \\
\hline Realizar oficinas para elaborar protocolos de regulação de acesso \\
\hline Planejamento das Redes de Atenção à Saúde - No de Ações: 1 (3\%) \\
\hline
\end{tabular}

Quadro 2. Categorização das ações educativas por temas

Fonte: Elaboração própria (2017).

Na categoria Formação Técnica/Profissional foram incluídas todas as propostas de formação que estivessem relacionadas ao aperfeiçoamento e qualificação de capacidades técnicas e/ou profissionais. Deste modo, ações que fizessem referência a procedimentos, rotinas e processos de trabalho ou a especialidades profissionais foram classificadas e 
contabilizadas nesta categoria. Foram identificadas 20 atividades com estas características, o que representa $60,6 \%$ de todas as ações analisadas.

Duas propostas previam a realização de atividades com o objetivo de elaboração ou formulação de novos fluxos, rotinas ou protocolos de trabalho. Tais ações foram agrupadas na categoria Elaboração de Protocolos, o que representa $6,1 \%$ do total de atividades propostas.

Cabe destacar a diferença entre estas duas categorias. Na categoria Formação Técnica/Profissional as ações educativas tratavam de protocolos e rotinas já existentes e utilizados rotineiramente. Na segunda categoria, Elaboração de Protocolos, só foram consideradas ações que previssem a elaboração de novas rotinas e protocolos que fossem resultantes das necessidades que emergiam a partir da conformação das RAS no território.

Dentre as atividades propostas, quatro estavam relacionadas ao uso de sistemas ou de tecnologias em saúde e foram classificadas na categoria Sistemas de Informação ou Telessaúde, representando $12,1 \%$ das ações analisadas.

Na categoria Estruturação das RAS foram incluídas as seis atividades $(18,2 \%)$ que tinham como tema central de trabalho a estruturação e organização das RAS.

Por último, na temática Planejamento das Redes de Atenção à Saúde foram agrupadas as atividades educativas que tivessem como proposta o planejamento das RAS, a partir do qual as redes seriam estruturadas e organizadas. Nesta categoria foi identificada apenas uma atividade, representando 3\% do total de atividades analisadas.

É importante explicar a diferença entre estas duas últimas categorias de análise: Estruturação das RAS e Planejamento das RAS. A primeira se refere a ações educativas propostas que tinham como foco de suas temáticas a preparação dos envolvidos para a implementação e operacionalização de serviços e atividades pertinentes a conformação da RAS na região. Na segunda estavam inseridas ações educativas que buscavam pensar o planejamento, do ponto de vista macroeconômico e político, ou seja, o foco era na formulação de estratégias políticas e orçamentárias das RAS nas regiões envolvidas. 
É importante destacar que estes dados demonstram as principais inquietações e demandas dos GC com relação às necessidades de formação de sua região. Nota-se uma ênfase acentuada em conteúdos técnicos e profissionalizantes, relacionados às especialidades terapêuticas, aos papéis profissionais atrelados aos serviços, cargos, funções e aprendizagem de sistemas e tecnologias. Isso pode ser confirmado ao analisarmos os temas propostos: $60,6 \%$ das ações propostas referiam-se a conteúdos técnicos e profissionais, tais como capacitação para diagnóstico de retinopatia ou treinamento para transporte sanitário e $12,1 \%$ se referiam às atividades voltadas ao aprendizado de sistemas e tecnologias.

Estas duas categorias juntas representam $72,7 \%$ do total das ações educativas analisadas, apontando um alinhamento com as atividades de EC visto que se propõem a "aproximar o vácuo existente entre a formação e a real necessidade do sistema de saúde", como afirma Pedroso (p.92). ${ }^{22}$ Neste sentido, tais propostas tratam de conteúdos técnicos com finalidade de capacitar os profissionais para exercer determinada rotina ou protocolo, operar determinado sistema ou equipamento, conhecer o fluxo dentro de uma linha de cuidado, etc. Sendo assim, apresentam características típicas de atividades de educação continuada (EC), que não se inserem no cotidiano do serviço, não abordam o processo de trabalho e não consideram o contexto e a realidade dos profissionais.

Cabe destacar que tais atividades estavam diretamente relacionadas aos eixos estruturantes do Projeto QualiSUS-Rede devendo, portanto, trazer assuntos que contemplassem tais dimensões. Este fato, atrelado às necessidades inerentes e pungentes de cada região, pode ter cerceado o leque de temas a serem propostos, reduzindo o número de temáticas a serem trabalhadas em cada subprojeto. No entanto, esta questão não se configura como um limitador de ações de EPS, visto que mesmo alguns temas propostos com características de EC poderiam ser ventilados e ofertados através de formatos mais em consonância com ações de EPS. 
Nesta perspectiva, as atividades educativas propostas foram também agrupadas de acordo com seus métodos de operacionalização, ou seja, de acordo com o formato proposto para execução de cada atividade: capacitação, treinamento, curso, oficina e seminário.

Tabela 1. Tipos de atividades educativas

\begin{tabular}{|l|c|c|}
\hline \multicolumn{1}{|c|}{ Denominação das Atividades Educativas } & N & \% \\
\hline Capacitação & 10 & 30,3 \\
\hline Curso & 10 & 30,3 \\
\hline Treinamento & 7 & 21,2 \\
\hline Oficina & 5 & 15,2 \\
\hline Seminário & 1 & 3 \\
\hline Total & $\mathbf{3 3}$ & $\mathbf{1 0 0}$ \\
\hline
\end{tabular}

Fonte: Elaboração própria (2017).

Os tipos de atividades educativas denotam características que as aproximam dos conceitos de educação permanente em saúde - EPS ou de educação continuada - EC. As concepções de EPS e de EC são distintas, no entanto, é extremamente importante não as rotular como antagônicas conceitualmente. Elas são processos de ensino-aprendizagem com especificidades em relação ao princípio educativo.

Diante do apresentado na Tabela 1, Marin $^{23}$ assinala a importância de uma reflexão crítica sobre cada tipo ou formato de atividade educativa, visto que o planejamento de uma ação de educação é orientado pelas concepções atribuídas aos termos utilizados. Então, mesmo que os termos empregados possam denotar equivalência, não se deve assumi-los como sinônimos.

A capacitação, que representa 30,3\% das ações propostas nos subprojetos analisados, é um conjunto de ações pedagógicas intrínsecas ao planejamento institucional com o objetivo de desenvolvimento contínuo de seus profissionais para o desempenho de suas atividades com eficiência e qualidade. ${ }^{24}$ 
Os cursos, também representando $30,3 \%$ dos dados analisados, indicam um processo sistemático e formal, orientado para conteúdos específicos, com ênfase teórica e comumente voltado para a atividade profissional visando formação ou qualificação.

As atividades de treinamento, que representaram 21,2\% neste estudo, são caracterizadas por ações que envolvem a transmissão de saberes específicos, orientadas para o trabalho, com foco no desenvolvimento e na melhoria de habilidades técnicas para atuação imediata das funções e cargos dos seus profissionais. ${ }^{24}$

As oficinas, que compreendem 15,2\% das ações educativas analisadas, constituem, de acordo com Candau, ${ }^{25}$ um ambiente de construção coletiva do conhecimento, de apreciação crítica e reflexiva da realidade, de um espaço de confrontação e de troca de saberes, com ênfase na socialização de experiências e experimentação de situações concretas, através de leituras, análises de casos, de sociodramas e expressões da cultura popular. O objetivo das oficinas é a produção de conhecimentos a partir de uma realidade concreta a fim de transformá-la. Permitem, portanto, o processo de ensino-aprendizagem com base na sensibilização, na compreensão, na reflexão, na análise, na ação e na avaliação, colocando os participantes como sujeitos ativos e agentes de transformação. ${ }^{26}$

Os seminários representam apenas 3\% das atividades propostas e se caracterizam pela interação ativa entre "professor, alunos expositores e audiência" (p. 80). ${ }^{27}$ Nesse sentido, quem conduz o seminário mantém um diálogo com os expectadores sobre um tema previamente sugerido pelo grupo ou pelo professor. Para Dolz et al. ${ }^{28, \text { p. } 218}$ os seminários são "um gênero textual público, relativamente formal e específico, no qual o expositor especialista dirige-se a um auditório, de maneira estruturada (explicitamente), para Ihe transmitir informações, descrever-Ihe ou explicar alguma coisa".

A literatura aponta a EC como uma educação baseada na transmissão de conhecimento, com ênfase no saber técnico-científico e tendo como foco as categorias profissionais na perspectiva de transformação da organização. ${ }^{10,14,29}$ A EC também contempla metodologias ativas de ensino-aprendizagem norteadas para transformações 
das práticas pedagógicas tradicionais, porém, tais conteúdos são direcionados e partem de decisões que apontam que tipo de qualificação ou de conteúdos os serviços e os profissionais precisam. Essas demandas por atividades educativas que preencham as necessidades de aquisição de conhecimentos específicos para o desempenho dos serviços fazem com que a EC seja desenvolvida geralmente no formato de cursos, treinamentos e capacitações. ${ }^{29}$ Seguindo este raciocínio, também as atividades operacionalizadas sob o formato de seminário se enquadram como ações de EC, visto suas características pedagógicas e a origem da escolha e decisão dos temas abordados.

Na EPS o processo de trabalho é adotado como elemento de investigação, de transformação e como cenário do processo de ensino-aprendizagem. É do cotidiano dos serviços que surgem as necessidades, que se identificam as lacunas de conhecimento e as atitudes explicativas para os problemas a serem trabalhados. As ações de EPS se constroem a partir das necessidades identificadas em contextos específicos no próprio processo de trabalho. Peduzzi et al. ${ }^{10}$ explicam que a EPS se fundamenta na aprendizagem significativa, onde a educação é concebida como estopim para a transformação das práticas no cotidiano dos serviços e no processo de trabalho. Sendo assim, as reflexões críticas sobre o trabalho, sobre os serviços e sobre os profissionais se processam na prática da EPS.

Diante deste contexto, as oficinas são atividades que buscam estimular a identificação de problemas relativos a um determinado contexto, por um determinado grupo inserido na realidade, para somente então serem trabalhadas internamente. Lembrando que as oficinas são comumente utilizadas para discussão de questões relativas ao processo de trabalho e são nelas que surgem os elementos para mudança das práticas. Assim, as oficinas podem ser caracterizadas como atividades de EPS, pois partem de indicações das necessidades concretas do processo de trabalho pelos próprios trabalhadores da saúde.

Ao considerar que as atividades de cursos, capacitações, treinamentos e seminários se apresentam com características de um processo de EC e que as oficinas dialogam mais proximamente com as propostas de EPS, foram encontrados os seguintes dados: $84,8 \%$ das 
propostas de ações educativas se caracterizam como EC enquanto apenas 15,2\% podem ser entendidas como ação de EPS.

Tabela 2. Ações educativas agrupadas como EC ou EPS de acordo com seus formatos

\begin{tabular}{c|c}
\multicolumn{1}{c|}{ EPS } & $\mathbf{1 5 , 2 0 \%}$ \\
\hline Ações & $N^{\circ}$ de ações \\
\hline OC & 5 \\
\hline Ações & $\mathbf{8 4 , 8 0 \%}$ \\
\hline Capacitação & $N^{\circ}$ de ações \\
\hline Curso & 10 \\
\hline Treinamento & 10 \\
\hline Seminário & 7 \\
\hline
\end{tabular}

Fonte: Elaboração própria (2017).

As análises realizadas sobre os temas abordados nos subprojetos e sobre os tipos de atividades propostas indicam certo desalinhamento entre o que é EPS e a compreensão dos profissionais gestores e técnicos do SUS.

Ainda que a recomendação inicial do Projeto QualiSUS-Rede fosse a elaboração de propostas de EPS nos subprojetos de cada região, os dados demonstram que a maior parte das ações educativas analisadas apresentavam características de EC, tanto no que se refere às temáticas abordadas, onde pelo menos $72,7 \%$ dos conteúdos propostos se relacionavam à formação e capacitação, como nos tipos de atividades propostas, onde apenas 15,2\% se enquadravam em propostas de EPS.

Embora a EC e a EPS sejam processos que podem ser complementares, eles se diferenciam em suas ações, objetivos e práticas. E tais diferenças não foram consideradas nas propostas estudadas, pois se observou que tais atividades estavam, em sua maioria, mais alinhadas às concepções EC, tanto no que se refere às temáticas propostas quanto aos formatos sugeridos para estas atividades. 
Importante ressaltar que no Manual Operacional do Projeto QualiSUS-Rede ${ }^{18}$ são apresentadas, a título de orientação para elaboração dos subprojetos, algumas intervenções aptas a receberem o investimento em questão. Estas proposições foram sugeridas de maneira a nortear as propostas dos subprojetos tendo como base os eixos estruturantes do Projeto QualiSUS-Rede (Quadro 3), ou seja, elas serviriam como exemplo para que cada subprojeto elaborasse suas próprias atividades.

\begin{tabular}{|c|c|c|}
\hline Nas & $S$ & $\mathrm{~S}$ \\
\hline $\begin{array}{l}\text { Estruturação e/ou } \\
\text { reestruturação física e } \\
\text { tecnológica de unidades que } \\
\text { compõem ou que venham a } \\
\text { compor a rede de serviços de } \\
\text { saúde de média complexidade } \\
\text { e de urgência e emergência } \\
\text { (Componente pré-hospitalar } \\
\text { fixo). } \\
\text { Processos } \\
\text { - Qualificação de processos } \\
\text { técnicos relacionados ao } \\
\text { cuidado: } \\
\text { Implementação de protocolos } \\
\text { clínicos, diretrizes terapêuticas } \\
\text { rotinas; } \\
\text { Qualificação de processos } \\
\text { clínicos tendo como referência } \\
\text { a saúde baseada em } \\
\text { evidências; } \\
\text { Melhoria dos procedimentos } \\
\text { relacionados ao acolhimento, } \\
\text { segurança dos pacientes e } \\
\text { biossegurança; } \\
\text { - Formulação e } \\
\text { implementação de } \\
\text { indicadores de avaliação e } \\
\text { de satisfação dos usuários; } \\
\text { Qualificação de processos } \\
\text { gerenciais: }\end{array}$ & $\begin{array}{l}\text { Governança } \\
\text { Definição e planejamento das } \\
\text { redes de atenção à saúde; } \\
\text { Desenvolvimento e implementação } \\
\text { de sistemas gerenciais; } \\
\text { Definição de responsabilidades } \\
\text { gestoras; } \\
\text { Definição de sistemas de } \\
\text { acompanhamento e avaliação; } \\
\text { Critérios e mecanismos para } \\
\text { acreditação das unidades que } \\
\text { compõem as redes; } \\
\text { Desenvolvimento de sistemas de } \\
\text { informação gerenciais; } \\
\text { Definição de instrumentos de } \\
\text { contratualização. } \\
\text { Desenvolvimento de } \\
\text { trabalhadores na prestação dos } \\
\text { serviços em saúde } \\
\text { Formação de gestores e gerentes } \\
\text { das redes. } \\
\text { Capacitação técnica para } \\
\text { desenvolvimento de funções } \\
\text { específicas nas redes. } \\
\text { Financiamento } \\
\text { Processos de análise econômica } \\
\text { (custo-benefício, custo efetividade); } \\
\text { Definição de mecanismos } \\
\text { inovadores de } \\
\text { gerenciamento/pagamento; } \\
\text { Desenvolvimento e implementação } \\
\text { de sistemas de custos das redes } \\
\text { de serviços, incluindo custos por } \\
\text { patologia ou grupos de } \\
\text { diagnósticos. }\end{array}$ & $\begin{array}{l}\text { Desenvolvimento de novos } \\
\text { instrumentos de gestão para } \\
\text { organização de redes de } \\
\text { atenção à saúde } \\
\text { Formas de pagamento; } \\
\text { Incentivos; } \\
\text { Contratualização. } \\
\text { Desenvolvimento e } \\
\text { qualificação de processos } \\
\text { gerenciais das SES para } \\
\text { implantação e organização das } \\
\text { redes de atenção à saúde } \\
\text { Sistemas de planejamento; } \\
\text { Sistemas de informação; } \\
\text { Sistemas de acompanhamento e } \\
\text { avaliação; } \\
\text { Desenvolvimento de modelos } \\
\text { inovadores de gestão de } \\
\text { unidades, redes e sistemas. } \\
\text { Educação Permanente - } \\
\text { Desenvolvimento de } \\
\text { trabalhadores na prestação dos } \\
\text { serviços em saúde } \\
\text { Capacitação e qualificação de } \\
\text { gestores municipais e estaduais e } \\
\text { de especialistas das SES e SMS } \\
\text { voltada à implementação das } \\
\text { linhas de cuidado e organização } \\
\text { das redes de atenção à saúde; } \\
\text { Qualificação e formação técnica } \\
\text { de nível médio para } \\
\text { trabalhadores e profissionais das } \\
\text { SES e SMS voltadas à } \\
\text { implementação das } \\
\text { de cuidado e organização das } \\
\text { redes de atenção à saúde; }\end{array}$ \\
\hline
\end{tabular}


incluindo custos por patologia

ou grupos de diagnósticos;

Desenvolvimento e

qualificação dos processos

administrativos e logísticos;

Implementação de processos

de gestão da qualidade;

Desenvolvimento e

qualificação de modelos de

compra e contratualização de

serviços;

Capacitação de trabalhadores

e profissionais para

qualificação dos processos

técnicos e gerenciais.

\section{Articulação na região de saúde \\ Desenvolvimento de mecanismos de referência e contra-referência; Definição de parâmetros para encaminhamento de pacientes entre os diferentes níveis de atenção.}

\section{Conformação/Modelagem de RAS}

Estruturação da APS;

Integração horizontal e vertical;

Modelagem da Rede de U\&E e de atenção a mulher e criança; Implantação de linhas de cuidado;

Definição e qualificação dos serviços de apoio diagnóstico e terapêutico:

a) Assistência farmacêutica;

b) Laboratórios de análises clínicas, serviços de diagnóstico de imagem, métodos gráficos e endoscópicos.

Definição e qualificação dos sistemas logísticos da rede:
a) Telecomunicação;
b) Centrais de regulação;
c) Transporte sanitário;
d) Sistemas de informação (implementação).

\section{Gestão do Trabalho}

Desenvolvimento de estratégias para dimensionar o quadro de pessoal necessário; Instalação de fóruns de negociação permanente entre gestores e trabalhadores;

Ajuste do protocolo de cessão de pessoal assinado na Mesa Nacional de Negociação Permanente do SUS.

Quadro 3. Possibilidades de Intervenção do Projeto QualiSUS-Rede nos Estados

Fonte: Manual Operacional do Projeto de Formação e Melhoria da Qualidade de Rede de Atenção à Saúde QualiSUS-Rede (grifos nossos). ${ }^{18,}$ p. 30

Como pode ser observado, as duas intervenções sombreadas no quadro 3 apresentam como atividades educativas ações de formação, de capacitação e de qualificação.

Adotando a análise realizada nos subprojetos, estas ações também apresentam atributos de EC. Ainda que se tratando de exemplos para elaboração dos subprojetos pelas regiões, tal fato demonstra que também existe dissonância entre a prerrogativa inicial do Projeto QualiSUS-Rede, que previa ações de EPS, e as atividades educativas esperadas dos subprojetos. Esta possível contradição reforça a hipótese de que não existe um 
entendimento claro dos conceitos EPS e EC entre os profissionais e gestores do SUS, mesmo dentre aqueles responsáveis pela elaboração de projetos, programas e políticas de saúde.

\section{Conclusão}

As propostas de EPS no Projeto QualiSUS-Rede não estão, em sua maioria, alinhadas aos conceitos de EPS e à PNEPS. Pelo previsto é identificada maior similaridade com a EC do que propriamente com a EPS, inclusive no próprio Manual Operacional do projeto, como foi possível observar. Aqui, contudo, aparece uma incongruência entre o que se tem de orientação e o que se necessitava de fato para atender aos objetivos do projeto QualiSUSRede.

Os dados analisados neste estudo indicam que existe uma distância entre as concepções de EPS como política pública de saúde e a compreensão dos profissionais e gestores do SUS. Lima, Albuquerque e Wenceslau ${ }^{30}$ confirmam essa confusão entre EC e EPS em seu estudo e acrescenta ainda a possibilidade de "uma utilização indiscriminada dos dois conceitos". ${ }^{30, \text { p. } 431}$

Tal constatação se reflete no planejamento dessas ações e pôde ser verificada nas propostas de ações educativas dos subprojetos QualiSUS-Rede que, inicialmente, preconizava atividades de EPS. Este resultado ressalta a importância de um trabalho de fortalecimento da Política Nacional de Educação Permanente em Saúde junto aos seus gestores e profissionais, não somente por se tratar de uma política, mas pelos benefícios inegáveis para os serviços, para os seus profissionais e para a sociedade.

O que se teve de fato foi que nenhuma das atividades educativas planejadas havia sido efetivamente realizada. Neste sentido, cabe apontar que uma análise das ações de educação executadas neste projeto se faz necessária como um possível desdobramento deste estudo. Tais dados podem agregar informações relevantes para a melhor 
compreensão dessa distância entre conceito e política e para compreensão das dificuldades de execução de ações reais de EPS no SUS.

Partindo dos resultados encontrados surge a seguinte questão para reflexão: em um Projeto como o QualiSUS-Rede, caberia a proposição somente de ações como EPS, visto que ambas (EPS e EC) são importantes para a organização das Redes de Atenção à Saúde? Embora neste estudo este não tenha sido um dos objetivos, é possível considerar que, dado o escopo e característica do Projeto QualiSUS-Rede, que procura induzir a organização das RAS a partir da formação e melhoria da qualidade dos serviços, as ações de EPS estariam mais alinhadas aos objetivos do QualiSUS-Rede. Isto porque as ações de EPS abordariam a temática por meio das demandas e necessidades do território, transformando o processo de trabalho a partir de necessidades inerentes aos serviços e às conformações das RAS.

Portanto, cabe enfatizar que a EPS e EC são complementares e não devem ser entendidas como antagônicas. Desta forma, estimular propostas concomitantes de atividades de EPS e EC nos projetos e programas do Ministério da Saúde, bem como fomentar o empoderamento e compreensão destas concepções e práticas pelos gestores e técnicos do SUS, se apresentam como estratégias que pode minimizar pequenos desalinhamentos entre os objetivos iniciais planejados e as ações implantadas e efetivamente executadas.

\section{Referências}

1. Brasil. Ministério da Saúde. Glossário temático: gestão do trabalho e da educação na saúde. 2a ed. Brasília, DF: Ministério da Saúde; 2012 [acesso 2014 maio 20]. Disponível em: http://bvsms.saude.gov.br/bvs/publicações/glossario_gestao_trabalho_2ed.pdf.

2. Schall VT, Struchiner M.Educação em saúde: novas perspectivas. Cad Saude Publica. 1999;15(Supl 2):S4-S6. http://dx.doi.org/10.1590/S0102-311X1999000600001 
3. Alves VS. Um modelo de educação em saúde para o Programa Saúde da Família: pela integralidade da atenção e reorientação do modelo assistencial. Interface Comun Saude Educ. 2005;9(16):39-52. http://dx.doi.org/10.1590/S1414-32832005000100004.

4. Rocha V, Schall VT, Lemos ES. A contribuição de um museu de ciências na formação de concepções sobre saúde de jovens visitantes. Interface Comun Saude Educ. 2010;14(32):183-96. http://dx.doi.org/10.1590/S1414-32832010000100015.

5. Alves $G G$, Aerts $D$. As práticas educativas em saúde e a estratégia saúde da família. Cienc Saude Colet. 2011;16(1):319-25. http://dx.doi.org/10.1590/S141381232011000100034

6. Morin E. A cabeça bem feita: repensar a reforma, reformar o pensamento. 7a ed. Rio de Janeiro: Bertrand Brasil; 2002.

7. Perrenoud P. Escola e cidadania: o papel da escola na formação para a democracia. Porto Alegre: Artmed; 2005.

8. Bordenave JD, Pereira AM. Estratégias ensino-aprendizagem. 28a ed. Petrópolis (RJ): Vozes; 2007.

9. Freire P. Educação e mudanças. 30a ed. Rio de Janeiro: Paz e Terra; 2007.

10. Peduzzi M, Guerra DAD, Braga CP, Lucena FS, Silva JAM. Atividades educativas de trabalhadores na atenção primária: concepções de educação permanente e de educação continuada em saúde presentes no cotidiano de Unidades Básicas de Saúde em São Paulo. Interface Comun Saude Educ. 2009;13(30):121-34. http://dx.doi.org/10.1590/S1414-32832009000300011.

11. Haddad J, Roschke MAC, Davini MC. Processo de trabajo y educacion permanente de personal de salud: reorientacion y tendencias en America Latina. Educ Med Salud. 1990;24(2):136-204.

12. Montanha D, Peduzzi M. Educação permanente em enfermagem: levantamento de necessidades e resultados esperados segundo a concepção dos trabalhadores. Rev Esc Enferm USP. 2010;44(3):597-604. http://dx.doi.org/10.1590/S0080-62342010000300007.

13. Ceccim RB. Educação permanente em saúde: desafio ambicioso e necessário. Interface Comun Saude Educ. 2005;9(16):161-77. http://dx.doi.org/10.1590/S141432832005000100013. 
14. Ceccim RB, Feuerwerker LCM. O Quadrilátero da formação para a área da saúde: ensino, gestão, atenção e controle social. Physis. 2004;14(1):41-65. http://dx.doi.org/10.1590/S0103-73312004000100004.

15. Brasil. Portaria $n^{\circ}$ 198/GM, de 13 de fevereiro de 2004. Institui a Política Nacional de Educação Permanente em Saúde como estratégia do Sistema Único de Saúde para a formação e o desenvolvimento de trabalhadores para o setor e dá outras providências. Diário Oficial da União, Brasília, DF (2004); Sec. 1:37-41.

16. Brasil. Portaria GM/MS n 1.996, de 20 de agosto de 2007. Dispõe sobre as diretrizes para a implementação da Política Nacional de Educação Permanente em Saúde. Diário Oficial da União, Brasília, DF (2007 ago 22);Sec. 1:34.

17. Brasil. Secretaria de Gestão do Trabalho e da Educação na Saúde (SGTES): políticas e ações. Brasília, DF: Ministério da Saúde; 2011 [acesso 2014 maio 19]. Disponível em: http://www.saude.es.gov.br/download/SGETS_Politicas_e_Acoes.pdf.

18. Brasil. Projeto de Formação e Melhoria da Qualidade de Rede de Atenção à Saúde QualiSUS-Rede. Brasília, DF: Ministério da Saúde; 2011.

19. Brasil. Portaria $n^{\circ} 396$, de 4 de março de 2011. Institui o Projeto de Formação e Melhoria da Qualidade de Rede de Atenção à Saúde (Quali-SUS-Rede) e suas diretrizes operacionais gerais. Diário Oficial da União, Brasília, DF (2011 maio 25);[Sec 1].

20. Brasil. Portaria GM/MS n 4.279, de 30 de dezembro de 2010: estabelece diretrizes para a organização da Rede de Atenção à Saúde no âmbito do Sistema Único de Saúde (SUS). Ministério da Saúde. Brasília, DF; 2010. Disponível em: <http://bvsms.saude.gov.br/bvs/saudelegis/gm/2010/prt4279_30_12_2010.html>. Acesso em: 04 abril 2014.

21. Brasil. Decreto $n^{\circ} 7.508 / G M$, de 28 de junho de 2011. Regulamenta a Lei no 8.080 , de 19 de setembro de 1990, para dispor sobre a organização do Sistema Único de Saúde SUS, o planejamento da saúde, a assistência à saúde e a articulação interfederativa, e dá outras providências. Diário Oficial da União, Brasília, DF (2011 jun 29) [acesso 2014 abr 4]. Disponível em: http://www.brasilsus.com.br/legislacoes/gm/108599-7508.html.

22. Pedroso VG. Aspectos conceituais sobre educação continuada e educação permanente em saúde. Mundo Saude (Impr.). 2005;29(1):88-93.

23. Marin AJ. Educação Continuada: introdução a uma análise de termos e concepções. Cad Cedes. 1995;(36):13-20. 
24. Chiavenato I. Gestão de pessoas. 2a ed. Rio de Janeiro: Campus; 2004.

25. Candau VM, Scavino SB, Marandino M, Maciel AG. Oficinas pedagógicas de direitos humanos. 2a ed. Petrópolis, RJ: Vozes; 1995.

26. Graciani MSS. Pedagogia social de rua. São Paulo: Cortez; 1997.

27. Goulart C. As práticas orais na escola: o seminário como objeto de ensino [dissertação]. Campinas: Universidade Estadual de Campinas; 2005.

28. Dolz J, Schneuwly B, De Pietro JF, Zahnd G. A exposição oral. In: Rojo R, Cordeiro GS, organizador, tradutor). Gêneros orais e escritos na escola. São Paulo: Mercado de Letras; 2004.

29. Batista KBC, Gonçalves OSJ. Formação dos profissionais de saúde para o SUS: significado e cuidado. Saude Soc. 2011;20(4):884-99. http://dx.doi.org/10.1590/S010412902011000400007

30. Lima SAV, Albuquerque PC, Wenceslau LD. Educação permanente em saúde segundo os profissionais da gestão de Recife, Pernambuco. Trab Educ Saude. 2014;12(2):425-41. http://dx.doi.org/10.1590/S1981-77462014000200012.

Patrícia Pássaro da Silva Toledo | ORCiD: 0000-0002-4013-7592

Doutoranda em Saúde Pública na ENSP/FIOCRUZ, Mestre em Ensino em Biociências e Saúde do Instituto Oswaldo Cruz - IOC/FIOCRUZ. Pesquisadora assistente no Laboratório de Avaliação de Situações Endêmicas Regionais - LASER/ENSP/FIOCRUZ.

\section{Marly Marques da Cruz}

Doutora em Saúde Pública pela ENSP/FIOCRUZ, Mestre em Saúde Pública pela ENSP/FIOCRUZ. Pesquisadora no Laboratório de Avaliação de Situações Endêmicas Regionais - LASER/ENSP/FIOCRUZ.

\section{Rafaela Barros Chagas de Souza}

Doutoranda em Saúde Pública na ENSP/FIOCRUZ, Mestre em Saúde Pública pela ENSP/FIOCRUZ. Pesquisadora assistente no Laboratório de Avaliação de Situações Endêmicas Regionais LASER/ENSP/FIOCRUZ. 